\title{
Protection of nigral dopaminergic neurons by AAV1 transduction with Rheb(S16H) against neurotoxic inflammation in vivo
}

Sehwan Kim ${ }^{1,11}$, Gyeong Joon Moon ${ }^{1,11}$, Yong-Seok Oh${ }^{2,11}$, Jungha Park ${ }^{1,11}$, Won-Ho Shin ${ }^{3}$, Jae Yeong Jeong ${ }^{4}$, Kwang Shik Choi ${ }^{1}$, Byung Kwan Jin ${ }^{4}$, Nikolai Kholodilov ${ }^{5}$, Robert E Burke, ${ }^{5,6}$, Hyung-Jun Kim ${ }^{7}$, Chang Man $\mathrm{Ha}^{7}$, Seok-Geun Lee ${ }^{8,9}$ and Sang Ryong Kim ${ }^{1,10}$

We recently reported that adeno-associated virus serotype 1 (AAV1) transduction of murine nigral dopaminergic (DA) neurons with constitutively active ras homolog enriched in brain with a mutation of serine to histidine at position 16 [Rheb(S16H)] induced the production of neurotrophic factors, resulting in neuroprotective effects on the nigrostriatal DA system in animal models of Parkinson's disease (PD). To further investigate whether AAV1-Rheb(S16H) transduction has neuroprotective potential against neurotoxic inflammation, which is known to be a potential event related to PD pathogenesis, we examined the effects of Rheb(S16H) expression in nigral DA neurons under a neurotoxic inflammatory environment induced by the endogenous microglial activator prothrombin kringle-2 (pKr-2). Our observations showed that Rheb(S16H) transduction played a role in the neuroprotection of the nigrostriatal DA system against $\mathrm{pKr}$-2-induced neurotoxic inflammation, even though there were similar

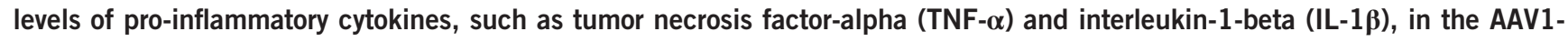
Rheb(S16H)-treated substantia nigra (SN) compared to the SN treated with pKr-2 alone; the neuroprotective effects may be mediated by the activation of neurotrophic signaling pathways following Rheb(S16H) transduction of nigral DA neurons. We conclude that AAV1-Rheb(S16H) transduction of neuronal populations to activate the production of neurotrophic factors and intracellular neurotrophic signaling pathways may offer promise for protecting adult neurons from extracellular neurotoxic inflammation.

Experimental \& Molecular Medicine (2018) 50, e440; doi:10.1038/emm.2017.261; published online 9 February 2018

\section{INTRODUCTION}

Parkinson's disease (PD) is the second most common neurodegenerative disease and is characterized by the loss of nigral dopaminergic (DA) neurons and a significant decrease in striatal dopamine levels. ${ }^{1,2}$ Although numerous types of drugs can temporarily relieve PD symptoms, there is no comprehensive therapy for $\mathrm{PD}$, and its pathogenic mechanism is largely unknown. ${ }^{3,4}$ However, neurotoxic inflammation is considered to be an important mechanism in the pathogenesis of $\mathrm{PD}$, and accumulating evidence indicates that activated microglia- mediated neurotoxic inflammation can exacerbate the loss of DA neurons and deteriorate the symptoms of the disease, suggesting that the control of activated microglia may be a useful strategy for neuroprotection in the adult brain. ${ }^{5-9}$

In PD brains, DA neurons in the substantia nigra $(\mathrm{SN})$ express decreased levels of glial cell line-derived neurotrophic factor (GDNF), a member of the transforming growth factor- $\beta$ family, and brain-derived neurotrophic factor (BDNF), a member of the neurotrophin family. ${ }^{10}$ Experimental results using GDNF ${ }^{11-13}$ and $\mathrm{BDNF}^{11,14}$ have consistently shown their

\footnotetext{
${ }^{1}$ School of Life Sciences, BK21 Plus KNU Creative BioResearch Group, Kyungpook National University, Daegu, Korea; ${ }^{2}$ Department of Brain-Cognitive Science, Daegu-Gyeongbuk Institute of Science and Technology, Daegu, Korea; ${ }^{3}$ Predictive Model Research Center, Korea Institute of Toxicology, Korea Research Institute of Chemical Technology, Daejeon, Korea; ${ }^{4}$ Department of Biochemistry and Molecular Biology, Department of Neuroscience Graduate School, School of Medicine, Kyung Hee University, Seoul, Korea; ${ }^{5}$ Department of Neurology, Columbia University, New York, NY, USA; ${ }^{6}$ Pathology and Cell Biology, Columbia University, New York, NY, USA; ${ }^{7}$ Department of Neural Development and Disease, Korea Brain Research Institute, Daegu, Korea; ${ }^{8}$ Department of Science in Korean Medicine, College of Korean Medicine, Seoul, Korea; ${ }^{9}$ KHU-KIST Department of Converging Science and Technology, Kyung Hee University, Seoul, Korea and ${ }^{10}$ Brain Science and Engineering Institute, Kyungpook National University, Daegu, Korea

${ }^{11}$ These authors contributed equally to this work.

Correspondence: Professor SR Kim, School of Life Sciences, BK21 Plus KNU Creative BioResearch Group, Kyungpook National University, Daegu 41566, Korea.
}

E-mail: srk75@knu.ac.kr

Received 6 March 2017; revised 11 July 2017; accepted 1 August 2017 
neuroprotective effects on DA neurons in animal models of PD. These results suggest that decreases in neurotrophic factors may be another important pathogenic mechanism involved in the loss of nigral DA neurons, ${ }^{10,15,16}$ even though a study reported that GDNF delivered by intracerebroventricular injection and intraputaminal infusion in patients with $\mathrm{PD}$ was ineffective for PD therapy, probably because of its limited penetration and distribution to the target brain areas. ${ }^{17}$ To clarify that the therapeutic potential of neurotrophic factors for PD depends on the sustained delivery of the appropriate amount to the target areas, we recently reported that adenoassociated virus 1 (AAV1) transduction of nigral DA neurons with a gene encoding the constitutively active form of ras homolog enriched in brain (Rheb) with a mutation of serine to histidine at position $16[\mathrm{Rheb}(\mathrm{S} 16 \mathrm{H})]$ induced a sustained production of neurotrophic factors, such as GDNF and BDNF, through activation of the mammalian target of rapamycin complex 1 (mTORC1), consequently resulting in the protection and restoration of the nigrostriatal DA projection in neurotoxin-treated animal models of PD. ${ }^{15,16,18,19}$ These observations demonstrate that the activation of neuronal mTORC1 induced by a specific gene delivery, such as Rheb(S16H), has neuroprotective effects against intracellular neurotoxicity.

There have been numerous reports showing that controlling the activation of microglia, which produce pro-inflammatory cytokines, may be a beneficial approach to protecting nigral DA neurons against neurodegeneration in the adult brain. ${ }^{8,9,20,21}$ However, there has been no report of a specific treatment that could induce neuroprotective effects without the control of neuroinflammation under a neurotoxic inflammatory environment in the adult brain, resulting in the protection of nigral DA neurons in vivo. Our previous observations suggested that activation of the intracellular neurotrophic signaling pathway by neuronal transduction with $\mathrm{Rheb}(\mathrm{S} 16 \mathrm{H})$ using a specific viral vector may be a useful strategy to impart the important ability of a protective potential against neurotoxicity to nigral DA neurons in the adult brain. ${ }^{15,22}$ Therefore, in the present study, we investigated whether Rheb $(\mathrm{S} 16 \mathrm{H})$ transduction of nigral DA neurons has a neuroprotective potential against neurotoxic inflammation induced by prothrombin kringle-2 $(\mathrm{pKr}-2)$, which was reported to be an endogenous microglial activator in a previous study, ${ }^{9}$ and whether neurotrophic factors, such as GDNF and BDNF, which can be produced by $\operatorname{Rheb}(\mathrm{S} 16 \mathrm{H})$ transduction into nigral DA neurons, ${ }^{15,16}$ contribute to the protective potential against $\mathrm{pKr}$-2-induced neurotoxic inflammation.

\section{MATERIALS AND METHODS}

\section{Production of AAV viral vectors}

All vectors used for these studies were AAV1 serotypes, as previously described. ${ }^{18,19}$ A plasmid carrying the Rheb gene was purchased from OriGene Technologies (Rockville, MD). Rheb DNA was amplified and modified to incorporate a FLAG-encoding sequence at the $3^{\prime}$-end by expanded long-template PCR (Roche, Indianapolis, IN, USA). Constitutively active Rheb $[\mathrm{Rheb}(\mathrm{S} 16 \mathrm{H})]$ was generated by use of the Phusion Site-directed Mutagenesis Kit of New England Biolabs
(Ipswich, MA, USA), integrated into the pGEM-T vector (Promega, San Luis Obispo, CA, USA), and then cloned into an AAV packaging construct that utilizes the chicken $\beta$-actin promoter and contains a $3^{\prime}$ WPRE (pBL). AAVs were produced by the University of North Carolina Vector Core, and the genomic titer was $3.6 \times 10^{12}$ viral genomes per ml. Enhanced green fluorescence protein (GFP), used as a control, was subcloned into the same viral backbone, and viral stocks were produced at a titer of $1 \times 10^{12}$ viral genomes per $\mathrm{ml}$.

\section{Institutional review of animal protocols}

Male C57BL/6 mice (8 weeks old, 23-25 g) were obtained from Daehan Biolink (Eumseong, Korea). The mice were housed in a controlled environment and were provided with food and water ad libitum. All surgical experiments were performed in accordance with the approved animal protocols and guidelines established by the Animal Care Committee of Kyungpook National University (No. KNU 2016-42).

\section{Intranigral AAV injection}

C57BL/6 mice were anesthetized by intraperitoneal injection of chloral hydrate (Sigma-Aldrich, St Louis, MO, USA; $360 \mathrm{mg} \mathrm{kg}^{-1}$ ) and placed in a stereotaxic frame (David Kopf Instrument, Tujunga, CA, USA). Each of the mice received a unilateral injection of AAV1-GFP as a control vector or AAV1-Rheb(S16H) using a 10- $\mu$ l Hamilton syringe (30S needle) attached to a syringe pump (KD Scientific, New Hope, $\mathrm{PA}, \mathrm{USA}$ ) into the right $\mathrm{SN}$ (anteroposterior (AP): $-3.5 \mathrm{~mm}$; mediolateral (ML): $-1.1 \mathrm{~mm}$; dorsoventral (DV): $-3.7 \mathrm{~mm}$, relative to bregma). A viral vector suspension in a volume of $2.0 \mu \mathrm{l}$ was injected at $0.1 \mu \mathrm{min}^{-1}$ over $20 \mathrm{~min}$. After the injection, the needle was left in place for an additional 5 min before being slowly retracted.

\section{Intranigral pKr-2 injection and neutralizing antibody injection}

Each animal received a unilateral injection of $\mathrm{pKr}-2$ alone (Haematologic Technologies, Inc., Essex Junction, VT, USA; $24 \mu \mathrm{g}$ in $2 \mu \mathrm{l}$ phosphate-buffered saline (PBS); Sigma-Aldrich) or in combination with neutralizing antibodies against GDNF and BDNF into the right $\mathrm{SN}$ (AP: $-3.2 \mathrm{~mm}$; ML: $-1.3 \mathrm{~mm}$; DV: $-4.0 \mathrm{~mm}$, relative to bregma) at a rate of $0.5 \mu \mathrm{min}^{-1}$. The following neutralizing antibodies were used: ${ }^{23}$ murine GDNF neutralizing antibody (R\&D Systems, Minneapolis, MN, USA; $200 \mathrm{ng}$ in $2 \mu \mathrm{l} \mathrm{PBS}$; Sigma-Aldrich) and murine BDNF neutralizing antibody (Santa Cruz Biotechnology, Santa Cruz, CA, USA; 200 ng in $2 \mu$ PBS; Sigma-Aldrich). After the injection, the needle was left in place for an additional 5 min before being slowly retracted. The animals were killed and analyzed at the indicated time points for each experiment.

\section{Immunohistochemical staining procedures}

Immunohistochemistry was performed as previously described, ${ }^{15,18}$ with some modifications for the present study. The animals were transcardially perfused with $4 \%$ paraformaldehyde in $0.1 \mathrm{M}$ phosphate buffer (PB). After post fixation and cryoprotection using a $30 \%$ sucrose solution, brain sections $(30-\mu \mathrm{m}$-thick) were processed for immunohistochemical staining. Briefly, sections were rinsed in $0.1 \mathrm{M}$ PBS and incubated with primary antibodies at $4{ }^{\circ} \mathrm{C}$ for 2 days. Tissues were rinsed with PBS- $0.5 \%$ bovine serum albumin, incubated at room temperature with appropriate biotinylated secondary antibodies, and then processed with an avidin-biotin complex kit (Vector Laboratories, Burlingame, CA, USA). The signal was detected by incubating sections in $0.5 \mathrm{mg} \mathrm{ml}^{-1} 3,3^{\prime}$-diaminobenzidine (DAB; Sigma-Aldrich) 
in $0.1 \mathrm{M}$ PB containing $0.003 \% \mathrm{H}_{2} \mathrm{O}_{2}$. The primary antibodies were rabbit anti-FLAG (1:3000; Sigma-Aldrich) and rabbit anti-tyrosine hydroxylase (TH; 1:2000; Pel-Freez, Brown Beer, WI, USA). Immunostained SN and striatum tissue samples were mounted on gelatincoated slides. SN tissue samples immunostained with the TH antibody were counterstained with $0.5 \%$ cresyl violet (Sigma-Aldrich) and then analyzed with a bright-field microscope (Axio Imager, Carl Zeiss, Göttingen, Germany).

Brain sections for double-immunofluorescence staining were rinsed and incubated for 2 days with one of the following pairs of antibodies: rabbit anti-TH (1:2000; Pel-Freez) and mouse anti-FLAG (1:3000; Sigma-Aldrich), mouse anti-glial fibrillary acidic protein (GFAP; 1:500; Sigma-Aldrich) and rabbit anti-FLAG (1:3000; Sigma-Aldrich), rabbit anti-ionized calcium-binding adapter molecule 1 (Ibal; 1:2000; Wako Pure Chemical Industries, Osaka, Japan) and mouse anti-FLAG (1:3000; Sigma-Aldrich), rabbit anti-Ibal (1:2000; Wako Pure Chemical Industries) and mouse anti-tumor necrosis factor-alpha (TNF- $\alpha$; 1:500; Santa Cruz Biotechnology), goat anti-Ibal (1:1000; Abcam, Cambridge, MA, USA) and rabbit anti-interleukin-1-beta (IL-1 $\beta$; 1:500; Santa Cruz Biotechnology), mouse anti-TH (1:200; R\&D Systems) and rabbit anti-phosphorylated-4E-BP1 (p-4E-BP1; 1:1000; Cell Signaling, Beverly, MA, USA), rabbit anti-TH (1:2000; Pel-Freez) and goat anti-GDNF (1:1000; R\&D Systems), and mouse anti-TH (1:200; R\&D Systems) and rabbit anti-BDNF (1:500; Santa Cruz Biotechnology) antibodies. After 2 days, sections were rinsed and incubated with Texas Red-conjugated anti-rabbit IgG, anti-mouse IgG, or anti-goat IgG (1:400; Vector Laboratories) and FITC-conjugated anti-mouse IgG, anti-rabbit IgG or anti-goat IgG (1:200; Jackson ImmunoResearch, West Grove, PA, USA) for $1 \mathrm{~h}$, and then they were washed and mounted with Vectashield mounting medium (Vector Laboratories). The stained sections were analyzed under a fluorescence microscope (Axio Imager).

\section{Western blot analysis}

The SN and striatum tissues were prepared from the animals at the indicated time points after injection of $\mathrm{pKr}-2$ as previously described with some modifications. ${ }^{9,15,18}$ Briefly, the tissues were homogenized and centrifuged at $4{ }^{\circ} \mathrm{C}$ for $15 \mathrm{~min}$ at $14000 \mathrm{~g}$. The supernatant was transferred to a fresh tube, and the concentration was determined using a bicinchoninic acid assay (BCA) kit (Bio-Rad Laboratories, Hercules, CA, USA). The samples were boiled at $100^{\circ} \mathrm{C}$ for $5 \mathrm{~min}$ before gel loading, and equal amounts of protein were loaded into each lane with the loading buffer. Proteins were separated using gel electrophoresis (Bio-Rad Laboratories) and electrophoretically transferred to polyvinylidene difluoride membranes (Millipore, Damstadt, Germany). The membranes were then incubated overnight at $4{ }^{\circ} \mathrm{C}$ with specific primary antibodies: rabbit anti-TH (1:2000; Pel-Freez), rabbit anti-Ibal (1:2000; Wako Pure Chemical Industries), mouse anti-TNF- $\alpha$ (1:500; Santa Cruz Biotechnology), rabbit anti-IL-1 $\beta$ (1:500; Santa Cruz Biotechnology), rabbit anti-4E-BP1 (1:1000; Cell Signaling), rabbit anti-p-4E-BP1 (1:1000; Cell Signaling), mouse antiGDNF (1:500; Santa Cruz Biotechnology), rabbit anti-BDNF (1:500; Santa Cruz Biotechnology), goat anti-tropomyosin receptor kinase B (TrkB; 1:1000; R\&D Systems), rabbit anti-phosphorylated TrkB (pTrkB; 1:1000; Millipore), anti-glial cell line-derived neurotrophic factor family receptor alpha 1 (GFR 1 1; 1:2000; Sigma-Aldrich), mouse anti-RET (1:1000; Santa Cruz Biotechnology), anti-phosphorylatedRET (p-RET; $1: 1000$; Sigma-Aldrich), and mouse anti- $\beta$-actin (1:1000; Santa Cruz Biotechnology). After washing with Tris-buffered saline with Tween-20 (TBS-T), the membranes were incubated with the appropriate secondary antibodies (Invitrogen, Camarillo, CA, USA) for $1 \mathrm{~h}$ at room temperature, and the blots were finally developed using enhanced chemiluminescence (ECL) western blot detection reagents (AbFrontier, Seoul, Korea). For semi-quantitative analyses, the density of the protein bands was measured using a computer imaging device and accompanying software (Fuji Film, Tokyo, Japan).

\section{Quantification of TH-positive neurons in the SN}

To determine the number of TH-positive neurons, both hemispheres of each mouse brain were analyzed as previously described with some modifications. ${ }^{8,18}$ Briefly, the entire SN from every section was identified as the region of interest, and the total number of THpositive neurons was counted using the optical fractionator's method with the Olympus Computer-Assisted Stereological Toolbox system version 2.1.4 (Olympus, Ballerup, Denmark). The number of cells in the SN was quantitatively expressed as a percentage of that in the contralateral control side.

\section{Quantitative determination of striatal TH immunostaining}

The whole striatum was identified as previously described, ${ }^{8,18}$ and the densitometric analysis was carried out using Science Lab 2001 Image Gauge (Fuji Film). TH-positive fiber innervation of the striatum was expressed quantitatively as the optical density on the ipsilateral lesioned side as a percentage of the optical density on the contralateral control side.

\section{Quantification of TH-positive axons in the medial forebrain bundle}

The number of TH-positive axons in the medial forebrain bundle (MFB) was analyzed as previously described. ${ }^{19}$ In brief, a horizontal section containing the A13 dopamine cell group, third ventricle and third ventricle recess was chosen for each animal. The region of interest was defined at low power to encompass the MFB. TH-positive fibers in each counting frame $(25 \mu \mathrm{m} \times 25 \mu \mathrm{m})$ were then determined by focusing down through the section under a $\times 100$ oil-immersion objective and were only counted when crossing both the top and bottom lines in the same frame.

\section{Dopamine and its metabolites in the striatum}

The measurement of striatal dopamine and its metabolites using reversed-phase high-performance liquid chromatography (HPLC) with an electrochemical detector was performed as previously described. ${ }^{9}$ Briefly, the brain tissues were homogenized and centrifuged, and then the supernatants were injected into an autosampler at $4{ }^{\circ} \mathrm{C}$ (Waters 717 plus autosampler) and eluted through a Sunfire C18 column $(4.6 \times 100 \mathrm{~mm} \times 5 \mu \mathrm{m}$; Waters Corporation, Milford, MA, USA) with a mobile phase for catecholamine analysis (Chromosystems, Munich, Germany). The peaks of dopamine and its metabolites, 3,4-dihydroxyphenylacetic acid (DOPAC) and homovanillic acid (HVA), were analyzed using an ESA Coulochem III electrochemical detector and integrated using a commercially available software program (ChemStation Software, Agilent Technologies, Santa Clara, CA, USA), and all samples were normalized for protein content as spectrophotometrically determined using the Pierce BCA protein assay kit (Thermo Scientific, Waltham, MA, USA).

\section{Rota-rod test}

Locomotor activity was evaluated using the rota-rod apparatus (30$\mathrm{mm}$ rod diameter; BS TechnoLab Inc., Seoul, Korea) as previously described. ${ }^{24}$ All mice were pre-trained on the rota-rod apparatus at a 
constant rate of 10 r.p.m. for $10 \mathrm{~min}$ once daily for three consecutive days. At 7 days after the $\mathrm{pKr}-2$ injection, the mice were placed on a constant accelerating rotating rod (4-40 r.p.m. within $5 \mathrm{~min}$ ). Two consecutive trials were performed at $60 \mathrm{~min}$ intervals. The latency to fall off the rota-rod was recorded.

\section{Statistical analysis}

All values are expressed as the mean \pm standard error of the mean (s.e. $\mathrm{m}$.). Multiple comparisons among groups were performed with oneway analysis of variance (ANOVA) followed by Tukey's post hoc analysis or independent sample $t$-test. All statistical analyses were performed using Sigma Stat software (Systat Software, San Leandro, CA, USA).

\section{RESULTS}

AAV1 transduction with $\mathrm{Rheb}(\mathrm{S} 16 \mathrm{H})$ in the $\mathrm{SN}$ in vivo

Mice were killed at 3 weeks after an intranigral injection of AAV1-GFP or AAV1-Rheb(S16H) to examine the viral transduction of nigral DA neurons, and the expression of target proteins was confirmed by observation of GFP expression and immunohistochemical staining for the FLAG epitope encoded in the Rheb $(\mathrm{S} 16 \mathrm{H})$ construct, respectively (Figure 1a and b). Consistent with our previous results, ${ }^{15,16,18}$ the doubleimmunofluorescence staining showed that GFP and FLAG were colocalized within TH-positive neurons (Figure 1b) but not glial cells (GFAP-positive astrocytes and Iba1-positive microglia) (Figure 1c). Similar to previous reports, ${ }^{15,16,18}$ GFP

a
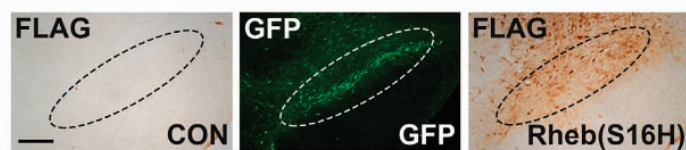

b

TH

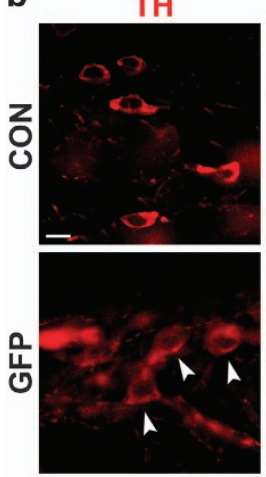

GFP/FLAG
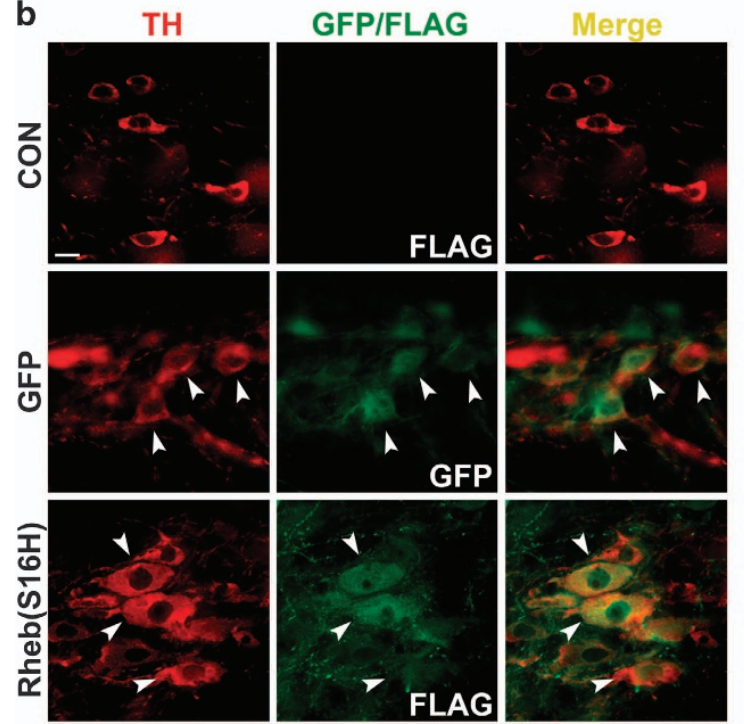

and FLAG were also observed in non-TH-positive neurons in the SN (Supplementary Figure S1), and Rheb $(\mathrm{S} 16 \mathrm{H})$ expression in nigral DA neurons induced morphological changes in neurons, indicated by arrow heads, such as increased neuronal size (Figure $1 \mathrm{~b})$, suggesting that $\mathrm{Rheb}(\mathrm{S} 16 \mathrm{H})$ administration had neurotrophic effects on nigral DA neurons.

$\mathrm{Rheb}(\mathrm{S} 16 \mathrm{H})$ expression in nigral DA neurons protects the nigrostriatal DA projection against $\mathrm{pKr}$-2-induced neurotoxicity

To examine whether nigral transduction with AAV1-Rheb $(\mathrm{S} 16 \mathrm{H})$ protects the nigrostriatal DA projection from neurotoxic inflammation, we unilaterally injected $\mathrm{pKr}-2$, which has been previously reported to be an endogenous microglial activator, $9,25-27$ into the SN at 3 weeks after AAV1-GFP and AAV1-Rheb(S16H) administration. The mice treated with $\mathrm{pKr}-$ 2-alone and $\mathrm{pKr}-2$ in the presence of GFP were used as controls for comparisons withRheb(S16H)-induced neuroprotection. At 7 days after $\mathrm{pKr}-2$ treatment, the brains were dissected, and the coronal sections were immunostained with the $\mathrm{TH}$ antibody. Our results showed that significant loss of TH-positive neurons and fibers was observed in the SN and striatum, respectively, of $\mathrm{pKr}$-2-treated mice and that the neurotoxicity of $\mathrm{pKr}-2$ treatment was similarly observed in the AAV1-GFP-treated mice (Figure 2a). When TH-positive neurons in the counting area of the ipsilateral SN were

C

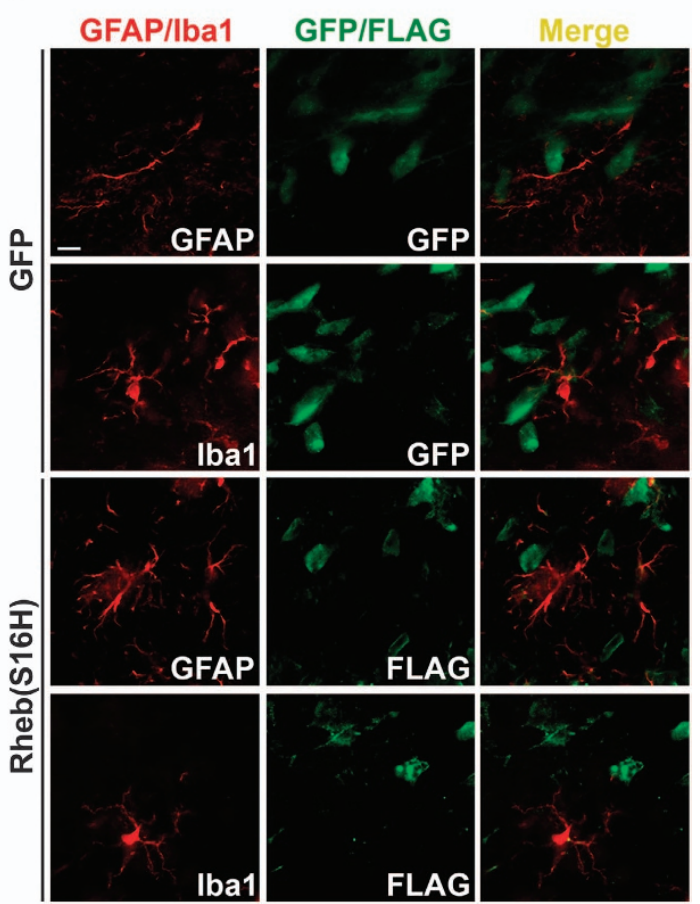

Figure 1 Transduction of nigral DA neurons with AAV1-GFP and AAV1-Rheb(S16H) in normal adult mice. (a) The expression of GFP and FLAG (brown reaction product) in the SN at 3 weeks after AAV1-GFP and AAV1-Rheb(S16H) administration for each viral injection. No expression of FLAG was observed in the non-injected control side (CON). The area inside the dotted lines is the substantia nigra pars compacta. Scale bar, $200 \mu \mathrm{m}$. (b) Double-immunofluorescence staining for TH (red) and GFP (green) and TH and FLAG (green) in the SN. Arrow heads indicate increased neuronal size induced by Rheb(S16H) expression in the nigral DA neurons. Scale bar, $20 \mu \mathrm{m}$. (c) Doubleimmunofluorescence staining for GFAP/Iba1 (red) and GFP (green), and GFAP/Iba1 and FLAG (green) in the SN. Scale bar, $20 \mu \mathrm{m}$. 
a
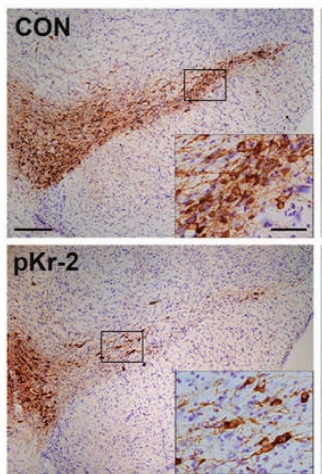

GFP + pKr-2

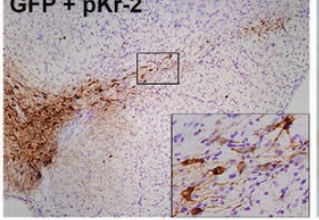

Rheb(S16H) + pKr-2
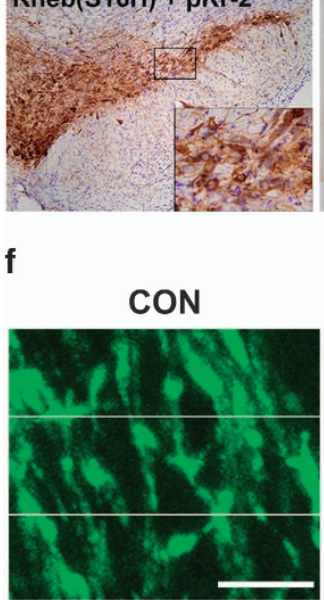

b

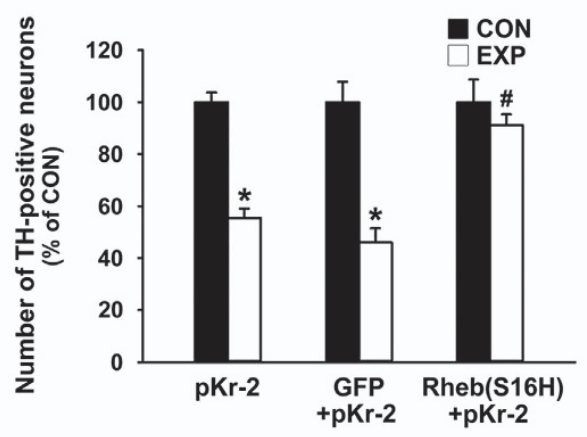

d

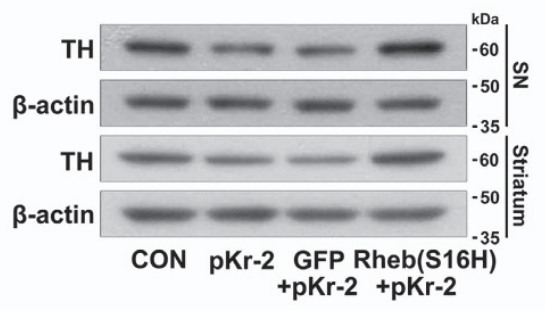

C
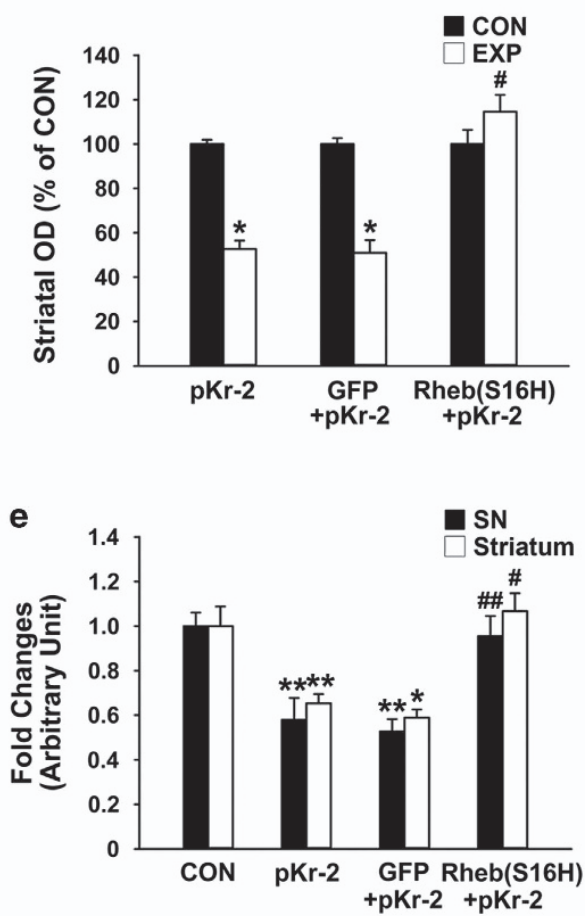

g

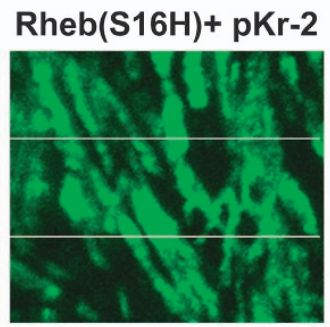

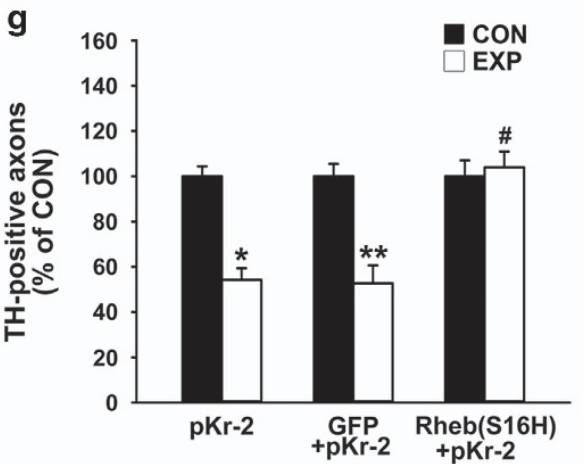

Figure 2 Rheb(S16H) protects the nigrostriatal DA pathway from pKr-2-induced neurotoxicity in vivo. (a) Representative coronal sections of the SN and striatum following TH immunostaining. CON, contralateral control side. Scale bars, 200 and $500 \mu \mathrm{m}$ in the SN and striatum, respectively. The small panels show higher magnifications of the SN. Scale bar, $50 \mu \mathrm{m}$. (b) Quantification of TH-positive neurons at 7 days after pKr-2 treatment. EXP, ipsilateral injected side. The histogram quantitatively shows TH-positive neurons in the counting area of the ipsilateral SN as a percentage of those in the contralateral controls. ${ }^{*} P<0.001$ vs contralateral controls, ${ }^{\#} P<0.01 \mathrm{vs}$ pKr-2 alone (oneway ANOVA and Tukey's post hoc analysis; $n=4$, each experimental group). All values are expressed as the mean \pm standard error of the mean (s.e.m.). (c) Quantitative determination of striatal TH immunostaining. The histogram shows the optical density of TH-positive fibers at 7 days after pKr-2 treatment. ${ }^{*} P<0.001$ vs contralateral controls, ${ }^{\#} P<0.001$ vs pKr-2 alone (one-way ANOVA and Tukey's post hoc analysis; $n=4$, each experimental group). All values are expressed as the mean \pm s.e.m. (d, e) Western blot analysis of TH expression at 7 days after pKr-2 treatment in the SN and striatum. The density of TH bands was normalized to the density of the $\beta$-actin band for each sample. ${ }^{*} P<0.01$ and ${ }^{* *} P<0.05$ vs contralateral controls, ${ }^{\#} P<0.01$ and ${ }^{\# \#} P<0.05$ vs pKr-2 alone (one-way ANOVA and Tukey's post hoc analysis; $n=3$, each experimental group). All values are expressed as the mean \pm s.e.m. (f) Representative horizontal sections of the medial forebrain bundle (MFB) following TH immunofluorescence staining. Scale bar, $10 \mu \mathrm{m}$. (g) Quantification of TH-positive axons at 7 days after injection of $\mathrm{pKr}$-2. The histogram quantitatively shows TH-positive axons in the counting area of the ipsilateral MFB as a percentage of those in the contralateral controls. ${ }^{*} P<0.001$ and ${ }^{* *} P<0.01$ vs contralateral controls, ${ }^{\#} P<0.001$ vs pKr-2 alone (one-way ANOVA and Tukey's post hoc analysis; $n=4$, between groups). All values are expressed as the mean \pm standard error of the mean (s.e.m.).

quantified and expressed as a percentage of those in the contralateral control, only $55 \%$ of DA neurons were preserved in the $\mathrm{SN}$ of the $\mathrm{pKr}$-2-treated mice (Figure $2 \mathrm{~b} ;{ }^{\star} P<0.001$ vs contralateral controls), whereas $91 \%$ of DA neurons were preserved in the presence of Rheb $(\mathrm{S} 16 \mathrm{H})$ (Figure $2 \mathrm{~b} ;{ }^{\#} \mathrm{P}<0.01$ vs $\mathrm{pKr}-2$ alone). These results suggest that $\mathrm{Rheb}(\mathrm{S} 16 \mathrm{H})$ is capable of inducing neuroprotection against the $\mathrm{pKr}$-2-induced death of nigral DA neurons. Similar to the effects in the SN, 53 
and $115 \%$ of $\mathrm{TH}$-positive fibers were observed in the striatum treated with $\mathrm{pKr}-2$ alone (Figure $2 \mathrm{c} ;{ }^{\star} P<0.001$ vs contralateral controls) and $\mathrm{pKr}-2$ in the presence of $\mathrm{Rheb}(\mathrm{S} 16 \mathrm{H}$ ) (Figure 2c; ${ }^{\#} \mathrm{P}<0.001$ vs $\mathrm{pKr}-2$ alone), respectively. Consistent with immunohistochemical staining results, western blot analysis showed that AAV1-Rheb(S16H) administration significantly preserved the levels of $\mathrm{TH}$ expression in both the $\mathrm{SN}$ and striatum compared to $\mathrm{pKr}-2$ alone (Figure $2 \mathrm{~d}$ and $\mathrm{e}$; ${ }^{\star} P<0.01$ and ${ }^{* *} P<0.05$ vs contralateral controls; ${ }^{\#} P<0.01$ and ${ }^{\#} P<0.05$ vs $\mathrm{pKr}-2$ alone). To further clarify if Rheb(S16H) induced neuroprotection, we examined the number of $\mathrm{TH}-$ positive axons in the MFB using the horizontal brain sections as previously described. ${ }^{19}$ As shown in Figure $2 \mathrm{f}$, the number of TH-positive axons in the MFB apparently decreased at 7 days after pKr-2-administration compared to that in the contralateral controls (Figure 2f). However, Rheb(S16H)-treated mice showed a significant preservation of $\mathrm{TH}$-positive axons against pKr-2-induced neurotoxicity (Figure 2f). The quantitative results, presented as a percentage of contralateral controls, showed that $54 \%$ and $52 \%$ of $\mathrm{TH}$-positive axons were observed in the MFB of $\mathrm{pKr}-2$ alone and AAV1-GFP-treated mice, respectively (Figure $2 \mathrm{~g} ;{ }^{\star} P<0.001$ and ${ }^{\star}{ }^{*} P<0.01$ vs contralateral controls). In contrast, $104 \%$ of TH-positive axons were observed in the MFB of Rheb(S16H)-treated mice compared to that in contralateral controls (Figure $2 \mathrm{~g}$ ). In addition to the histological protection, we further investigated the levels of dopamine and its metabolites, DOPAC and HVA, in the striatum using reversed-phase HPLC with an electrochemical detector. Similar to our previous study, ${ }^{9}$ treatment with $\mathrm{pKr}-2$ alone caused a significant reduction in dopamine levels in the striatum by $49 \%$ of the levels in the contralateral controls (Figure 3a; ${ }^{\star} P<0.001$ ), and decreases in both DOPAC and HVA were also observed in pKr-2-treated mice (Figure $3 \mathrm{~b}$ and $c ;{ }^{\star} P<0.001$ and ${ }^{*}{ }^{*} P<0.01$ vs contralateral controls). However, the levels of dopamine and its metabolites were significantly preserved in the striatum of Rheb(S16H)-treated mice compared to the levels observed in mice treated with $\mathrm{pKr}-2$ alone (Figure $3 \mathrm{a}-\mathrm{c}$; ${ }^{\#} \mathrm{P}<0.001$ vs $\mathrm{pKr}-2$ alone). Similar to the HPLC results, the analyses of the rota-rod behavioral tests showed that the pKr-2-injected mice exhibited a significant decrease in locomotor activity compared to control mice (Figure 3d; $\left.{ }^{\star} P<0.05\right)$. However, Rheb $(\mathrm{S} 16 \mathrm{H})$-treated mice showed a significant protection of locomotor activity compared to mice treated with $\mathrm{pKr}-2$ alone (Figure $3 \mathrm{~d}$; ${ }^{\#} \mathrm{P}<0.05$ vs $\mathrm{pKr}-2$ alone). These results demonstrated that AAV1-Rheb(S16H) transduction could induce a functional preservation of locomotor activity through protection of the nigrostriatal DA projections from $\mathrm{pKr}$-2-induced neurotoxicity.

\section{Preservation of GDNF and BDNF induced by Rheb(S16H) administration under the $\mathrm{pKr}$-2-induced neurotoxic inflammatory environment}

To investigate whether the induction of Rheb(S16H) expression in DA neurons affects $\mathrm{pKr}$-2-induced microglial activation, which induces neurotoxic inflammation in the nigrostriatal DA system, ${ }^{9,25-27}$ we evaluated the levels of activated microglia-produced pro-inflammatory cytokines, such as TNF- $\alpha$ and IL-1 $\beta$, in the SN. As shown in the double-immunofluorescence staining, there were apparent increases in TNF- $\alpha$ and IL-1 $\beta$ expression levels within Iba1positive microglia in the $\mathrm{SN}$ at 1 day after $\mathrm{pKr}-2$ treatment (Figure 4a). Consistent with the double-immunofluorescence staining results, western blot analysis at post-lesion day 1 showed significant increases in the levels of TNF- $\alpha$ and IL-1 $\beta$ in the pKr-2-treated SN compared to those in the contralateral controls (Figure $4 \mathrm{~b}$ and $\mathrm{c} ;{ }^{\star} P<0.01$ and ${ }^{*} P<00.05$ ). In the presence of Rheb $(\mathrm{S} 16 \mathrm{H})$, we found that there was no change in the $\mathrm{pKr}-2$-induced increase in TNF- $\alpha$ or IL- $1 \beta$ levels compared to that in the presence of $\mathrm{pKr}-2$ alone (Figure $4 \mathrm{~b}$ and c). These results indicate that neuroinflammatory responses induced by pKr-2-activated microglia were not affected by AAV1-Rheb (S16H) administration.

Recently, we reported that AAV1-Rheb(S16H) transduction could induce the sustained production of neurotrophic factors, such as GDNF and BDNF, through the activation of mTORC1 in nigral DA neurons in vivo and that this upregulation could contribute to the protection of nigrostriatal DA projections in neurotoxin-treated animal models of PD. ${ }^{15,18}$ To investigate whether Rheb(S16H)-transduced DA neurons could produce GDNF and BDNF as neuroprotective reagents under the neurotoxic inflammatory environment induced by $\mathrm{pKr}-2$ treatment, we next observed the expression levels of $\mathrm{p}-4 \mathrm{E}-$ BP1, indicating mTORC1 activation, and GDNF and BDNF following $\mathrm{pKr}-2$ treatment in the absence or presence of Rheb $(\mathrm{S} 16 \mathrm{H})$. As shown by the double-immunofluorescence staining, there were apparent increases in p-4E-BP1, GDNF and BDNF levels within TH-positive DA neurons at 3 weeks after AAV1$\mathrm{Rheb}(\mathrm{S} 16 \mathrm{H})$ administration in the SN (Figure 5a). To investigate whether the upregulation of $\mathrm{p}-4 \mathrm{E}-\mathrm{BP} 1, \mathrm{GDNF}$ and $\mathrm{BDNF}$ by $\mathrm{AAV} 1-\mathrm{Rheb}(\mathrm{S} 16 \mathrm{H})$ administration is preserved in the pKr-2-treated SN, we evaluated the levels with western blot analysis at 1day and 7 days after $\mathrm{pKr}-2$ treatment. Our results showed that the increased levels of p-4E-BP1, GDNF and BDNF were significantly preserved in the SN treated with $\mathrm{pKr}$ 2 in the presence of $\mathrm{Rheb}(\mathrm{S} 16 \mathrm{H})$ at post-lesion day 1 compared to the levels in the contralateral controls (Figure $5 \mathrm{~b} ;{ }^{\star} P<0.001$ and ${ }^{* *} P<0.01$ vs contralateral controls) and that there was no significant change in $\mathrm{p}-4 \mathrm{E}-\mathrm{BP} 1, \mathrm{GDNF}$ or BDNF expression in the $\mathrm{SN}$ treated with $\mathrm{pKr}-2$ alone and that treated with $\mathrm{pKr}-2$ in the presence of GFP compared to the levels in the contralateral controls (Figure 5b). Moreover, the levels of p-4E-BP1, GDNF and BDNF were substantially preserved in the SN treated with pKr-2 in the presence of $\mathrm{Rheb}(\mathrm{S} 16 \mathrm{H})$ at post-lesion day 7, when significant neurotoxicity by $\mathrm{pKr}-2$ administration appeared, compared to the levels in contralateral controls (Figure $5 c$; ${ }^{\# \#} P<0.01$ and ${ }^{\# \#} P<0.05$ vs $p K r-2$ alone), even though there were significant decreases in the levels of $\mathrm{p}-4 \mathrm{E}-$ $\mathrm{BP} 1, \mathrm{GDNF}$ and BDNF in the SN treated with $\mathrm{pKr}-2$ alone and that treated with $\mathrm{pKr}-2$ in the presence of GFP at post-lesion day 7 compared to the levels in contralateral controls (Figure $5 c ;{ }^{*} P<0.001,{ }^{*} P<0.01$ and ${ }^{* *} P<0.05$ ). Thus, these results suggest that the productive activities of neurotrophic 

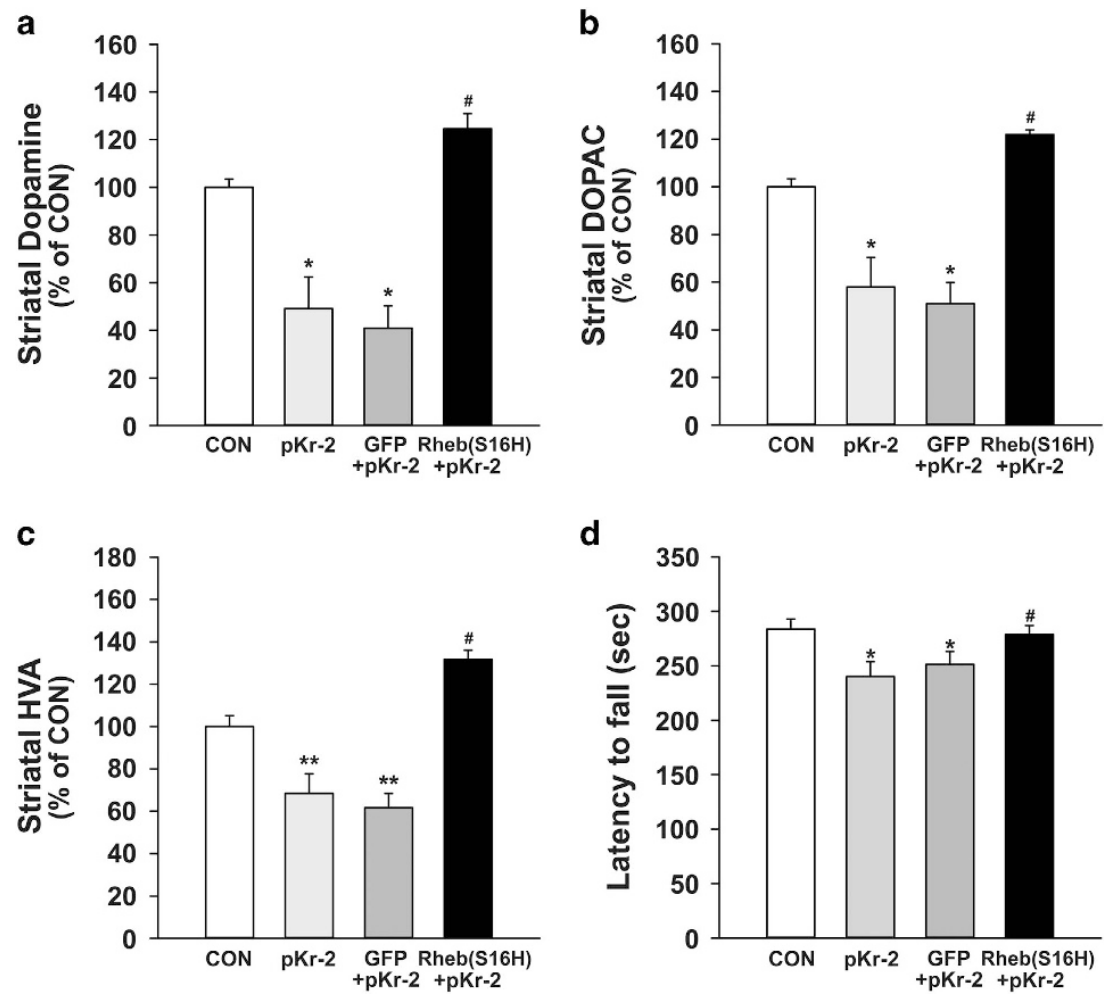

Figure 3 The functional preservation of Rheb(S16H) against the pKr-2-induced neurotoxic events in vivo. (a-c) The amounts of striatal dopamine and its metabolites, such as DOPAC and HVA, were measured by HPLC, and the level was quantitatively expressed as a percentage of the level in contralateral controls. ${ }^{*} P<0.001$ and ${ }^{* *} P<0.01$ vs contralateral controls, ${ }^{\#} P<0.001$ vs pKr-2 alone (one-way ANOVA and Tukey's post hoc analysis; $n=5$, each experimental group). All values are expressed as the mean \pm standard error of the mean (s.e.m.). (d) Locomotor activity was measured with the rota-rod test. ${ }^{*} P<0.05$ vs control mice, ${ }^{\#} P<0.05$ vs pKr-2 alone ( $t$-test analysis; $n=8$, each experimental group). All values are expressed as the mean \pm s.e.m.

factors following transduction with AAV1-Rheb(S16H) may be preserved under the neurotoxic inflammatory environment induced by $\mathrm{pKr}-2$ treatment in the SN of the adult brain.

\section{Upregulation of GDNF and BDNF following AAV1-Rheb} $(\mathrm{S} 16 \mathrm{H})$ administration contributes to the neuroprotection against $\mathrm{pKr}$-2-induced neurotoxicity

To investigate whether GDNF and BDNF increased by Rheb $(\mathrm{S} 16 \mathrm{H})$ expression in nigral DA neurons contribute to the neuroprotection of the nigrostriatal DA projection against the $\mathrm{pKr}$-2-induced neurotoxicity, we unilaterally injected $\mathrm{pKr}-2$ alone or in combination with neutralizing antibodies against GDNF and BDNF, which previously showed no alteration in the number of TH-positive neurons by treatment with neutralizing antibodies alone ${ }^{15}$ into the $\mathrm{SN}$ in the presence of $\mathrm{Rheb}(\mathrm{S} 16 \mathrm{H})$. The results of the immunohistochemical staining for $\mathrm{TH}$ showed that the neuroprotective effects of $\mathrm{Rheb}(\mathrm{S} 16 \mathrm{H})$ against $\mathrm{pKr}$-2-induced neurotoxicity, which preserved $90 \%$ of TH-positive neurons and $113 \%$ of TH-positive fibers in the $\mathrm{SN}$ and striatum, respectively, compared to contralateral controls(Figure $6 \mathrm{a}$ and b), were apparently attenuated by treatment with neutralizing antibodies against GDNF and BDNF (Figure 6a). In the presence of Rheb(S16H), when the TH-positive neurons in the counting area of the ipsilateral SN were quantified and expressed as a percentage of that in the contralateral controls, $52 \%$ and $47 \%$ of the THpositive neurons in the $\mathrm{SN}$ treated with $\mathrm{pKr}-2$ and anti-GDNF and the SN treated with $\mathrm{pKr}-2$ and anti-BDNF, respectively, were preserved [Figure $6 \mathrm{~b} ;{ }^{\star} P<0.001$ vs contralateral controls; ${ }^{\#} P<0.01$ vs $\mathrm{pKr}-2$ alone in the presence of $\left.\mathrm{Rheb}(\mathrm{S} 16 \mathrm{H})\right]$. Consistently, $40 \%$ and $47 \%$ of the $\mathrm{TH}$-positive fibers were preserved in the striatum by treatment with anti-GDNF and anti-BDNF antibodies, respectively (Figure $6 c ;{ }^{*} P<0.01$, ${ }^{*} P<0.001$ vs contralateral controls; ${ }^{\# \#} P<0.01,{ }^{\#} P<0.001$ vs $\mathrm{pKr}-2$ alone in the presence of $\mathrm{Rheb}(\mathrm{S} 16 \mathrm{H}))$. Moreover, cotreatment with anti-BDNF and anti-GDNF resulted in a synergistic inhibition of the neuroprotection of TH-positive neurons in the $\mathrm{SN}$ of Rheb(S16H)-treated mice (Figure $6 \mathrm{~b}$ ).

Furthermore, to clarify whether the increases in BDNF and GDNF following Rheb(S16H) administration contributed to the activation of the neurotrophic signaling pathway, suggesting an intensification of the Rheb(S16H)-induced neuroprotection, we examined the changes in the levels of neurotropic factors and their receptors, such as TrkB, p-TrkB, GFR $\alpha 1$, RET and p-RET, following treatment with anti-BDNF and anti-GDNF with western blot analysis. As shown in Supplementary Figure S2, Rheb $(\mathrm{S} 16 \mathrm{H})$ transduction significantly increased both the levels of BDNF and GDNF and the levels of TrkB, p-TrkB, GFR $\alpha 1$, RET and p-RET compared to the levels in the contralateral controls (Supplementary Figure S2c and d). The 
a
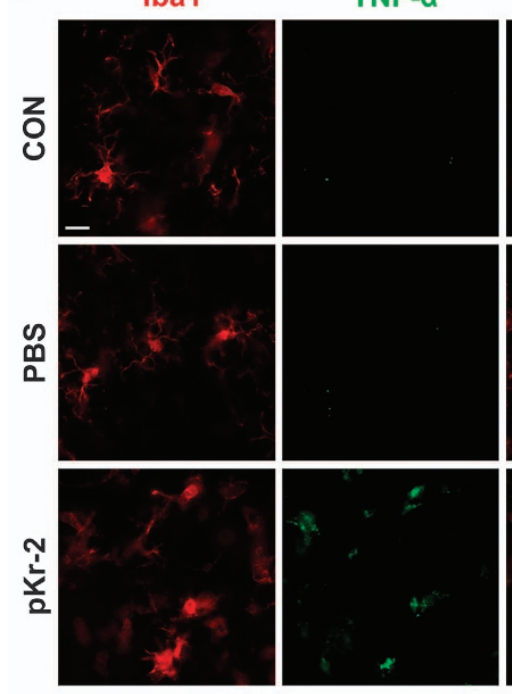

b

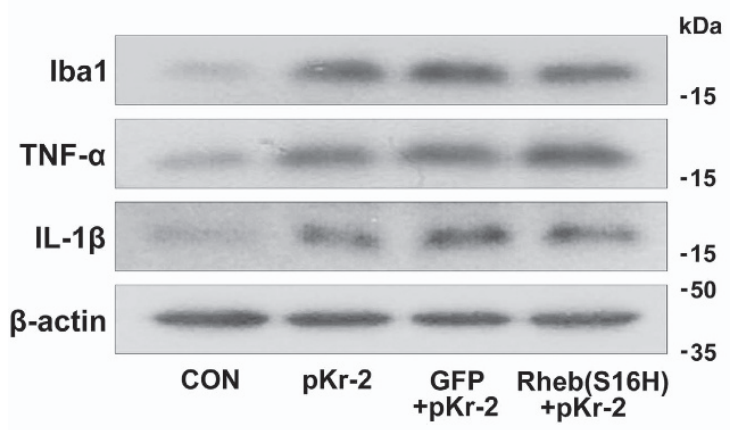

Merge
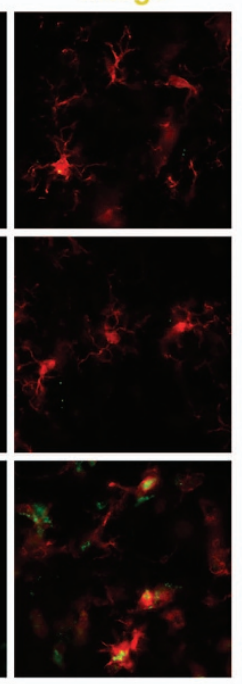
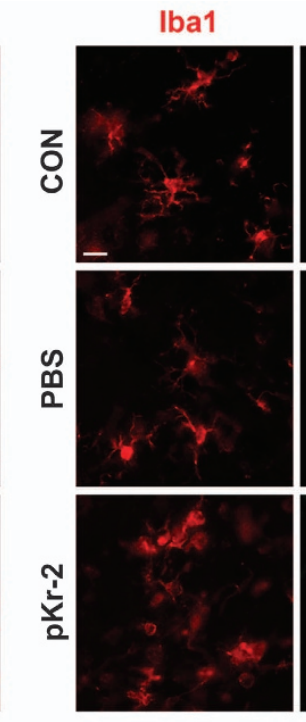

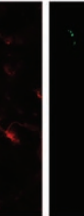

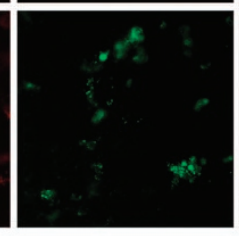

c

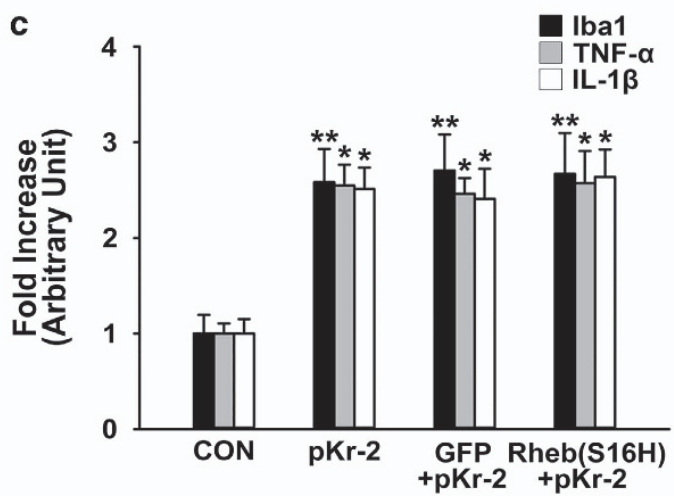

Figure 4 Increase in neuroinflammatory responses induced by pKr-2 treatment in the SN. (a) Double-immunofluorescence staining for TNF- $\alpha$ and IL-1 $\beta$ within Iba1-positive microglia in the SN at 1 day after pKr-2 treatment. CON, contralateral control side. Scale bars, $20 \mu \mathrm{m}$. (b, c) Western blot analysis of Iba1, TNF- $\alpha$ and IL-1 $\beta$ at 1 day after pKr-2 treatment in the SN. The density of Iba1, TNF- $\alpha$, and IL-1 $\beta$ bands was normalized to the density of the $\beta$-actin band for each sample. ${ }^{*} P<0.01$ and ${ }^{* *} P<0.05$ vs contralateral controls (one-way ANOVA and Tukey's post hoc analysis; $n=4$, each experimental group). All values are expressed as the mean \pm s.e.m.

levels of BDNF and p-TrkB were decreased in the mice treated with anti-BDNF alone, and the levels of GDNF and p-RET were decreased in the mice treated with anti-GDNF alone. Moreover, co-treatment with anti-BDNF and anti-GDNF showed synergetic effects controlling the Rheb(S16H)-induced increase of these factors (Supplementary Figure S2c and d), which may result in the inhibition of the Rheb(S16H)-induced neuroprotection in the SN (Figure 6b). Loss of TH-positive neurons was not observed with treatment with the neutralizing antibodies alone (Supplementary Figure S3). Similar to the inhibitory effects of anti-BDNF and anti-GDNF on the levels of trophic factors, the activation of mTORC1 following Rheb $(\mathrm{S} 16 \mathrm{H})$ administration was significantly attenuated by treatment with neutralizing antibodies against BDNF and GDNF (Supplementary Figure S2a). Taken together, our results demonstrate that Rheb(S16H) expression in nigral DA neurons can have protective effects against $\mathrm{pKr}$-2-induced neurotoxic inflammation and that the increase in neurotrophic factors such as GDNF and BDNF contributes to the intensification of the Rheb(S16H)-induced beneficial effects in the adult brain.

\section{DISCUSSION}

Although the cause of PD remains elusive, accumulating evidence suggests that neuroinflammatory responses are crucial for the initiation and progression of PD and that microglia, which are the resident immune cells in the brain and are observed in the SN of patients with PD and animal models of $\mathrm{PD}$, are important mediators of brain inflammation, which can trigger neurotoxic events. ${ }^{6,28-31}$ In the healthy brain, microglia are morphologically characterized by small cell bodies with thin and ramified processes, signifying low levels of inflammatory molecules associated with immune functions. ${ }^{5-8}$ However, under neuropathological conditions, activated microglia produce a spectrum of potentially neurotoxic molecules that contribute to the death of DA neurons. ${ }^{5-8}$ Recently, we also reported that upregulation of $\mathrm{pKr}-2$, which is an endogenous microglial activator, could induce the degeneration of nigrostriatal DA projections in the adult brain through production of pro-inflammatory cytokines such as TNF- $\alpha$ and IL- $1 \beta$ from activated microglia. ${ }^{9,25-27}$ These reports suggest that the control of microglial activation, which induces neurotoxic events, may 
a

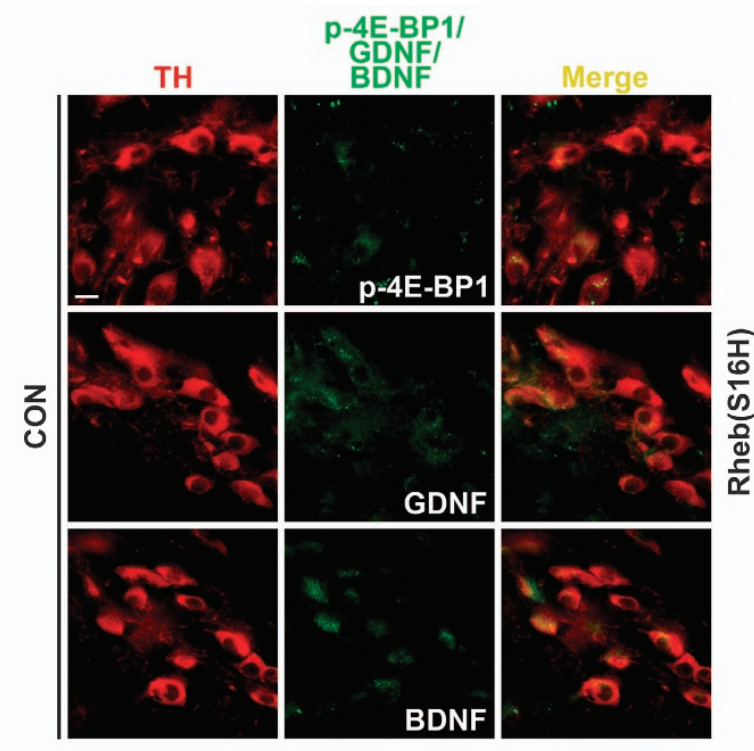

b

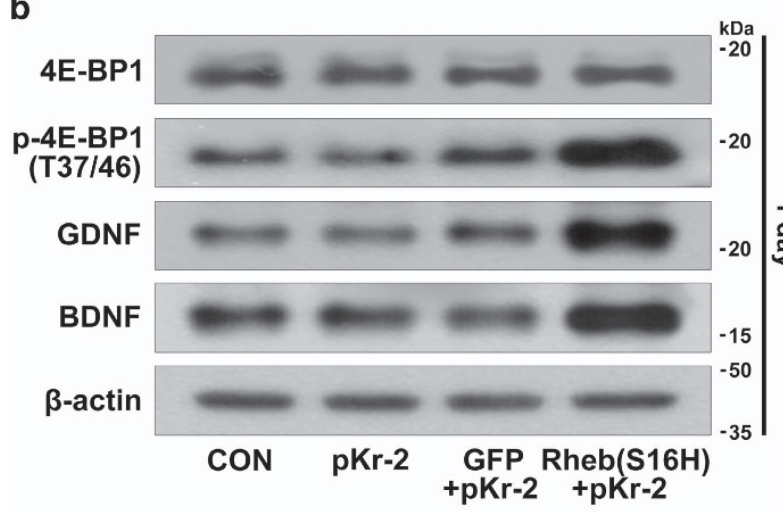

C

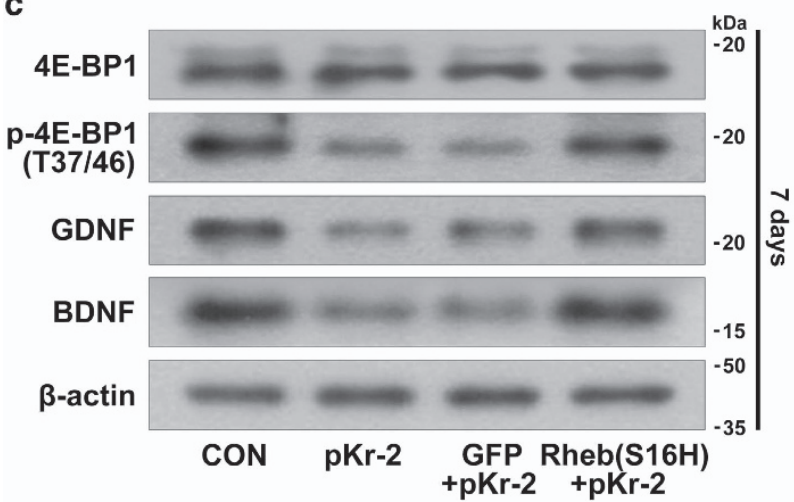

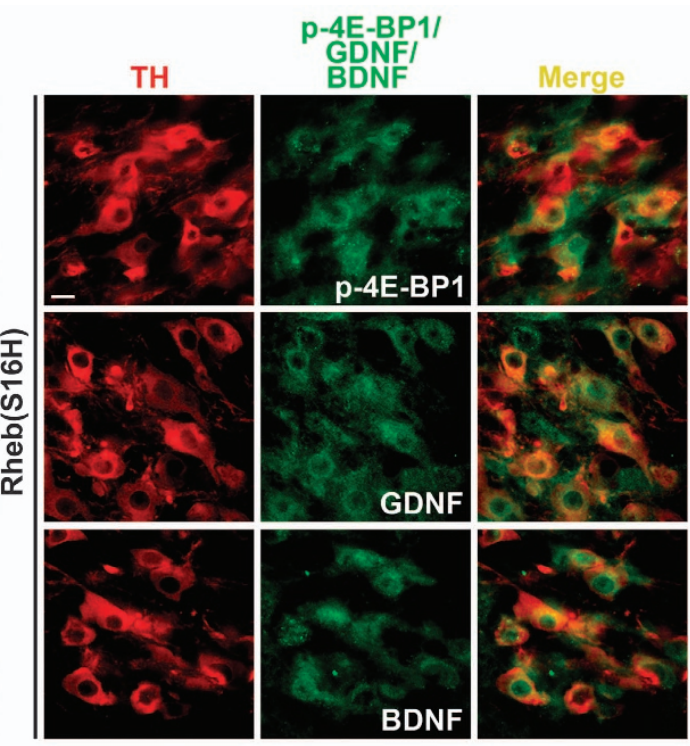
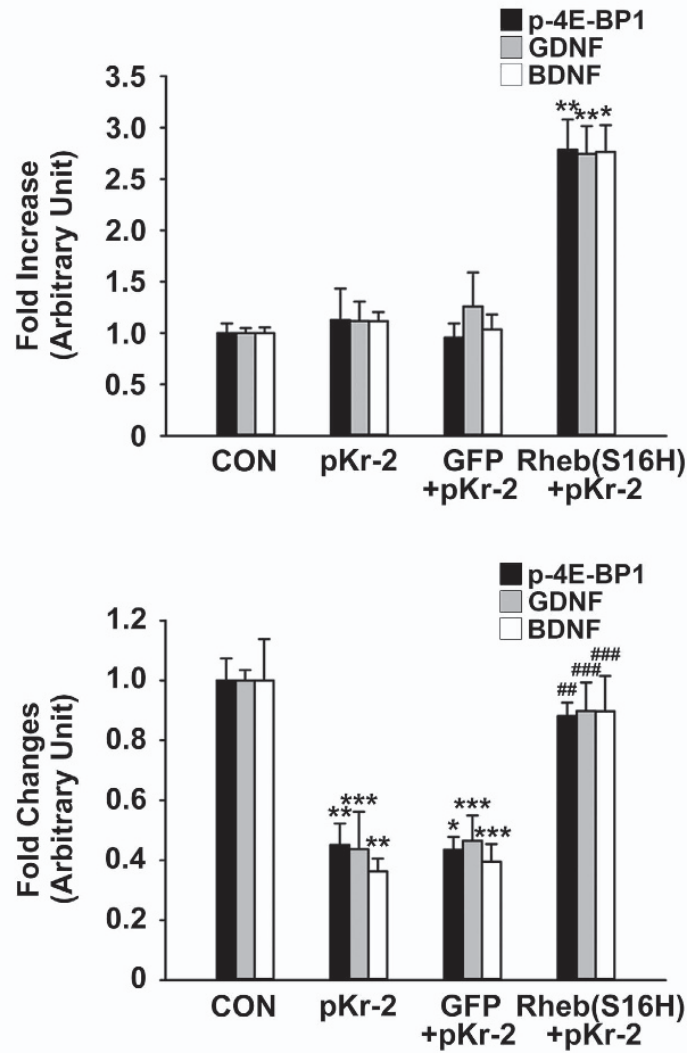

Figure 5 Preservation of mTORC1 activation and neurotrophic factors induced by Rheb(S16H) in neuroinflammatory conditions. (a) Double-immunofluorescence staining for TH (red) and p-4E-BP1 (green), TH and GDNF (green), and TH and BDNF (green) in the SN at 3 weeks after AAV1-Rheb(S16H) administration. CON, contralateral control side. Scale bars, $20 \mu \mathrm{m}$. (b) Western blot analysis of 4EBP1, p-4E-BP1, GDNF and BDNF in the SN at 1 day after pKr-2 treatment. The density of p-4E-BP1, GDNF, and BDNF bands was normalized to the density of the $\beta$-actin band for each sample. ${ }^{*} P<0.001$ and ${ }^{* *} P<0.01$ vs contralateral controls, pKr-2 alone and pKr-2 in the presence of GFP (one-way ANOVA and Tukey's post hoc analysis; $n=4$, each experimental group). All values are expressed as the mean \pm s.e.m. (c) Western blot analysis of $4 \mathrm{E}-\mathrm{BP} 1, \mathrm{p}-4 \mathrm{E}-\mathrm{BP} 1, \mathrm{GDNF}$, and BDNF in the SN at 7 days after pKr-2 treatment. The density of $\mathrm{p}-4 \mathrm{E}-\mathrm{BP} 1, \mathrm{GDNF}$, and BDNF bands was normalized to the density of the $\beta$-actin band for each sample. ${ }^{*} P<0.001,{ }^{* *} P<0.01$ and

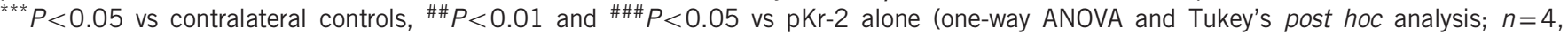
each experimental group). All values are expressed as the mean \pm s.e.m. 
a

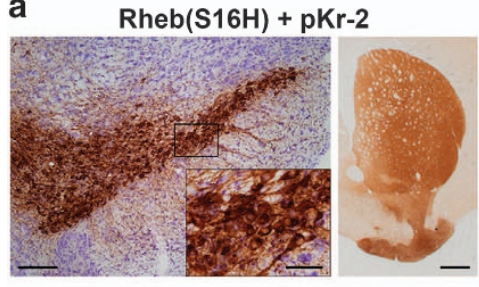

Rheb(S16H) + pKr-2 + GDNF NA

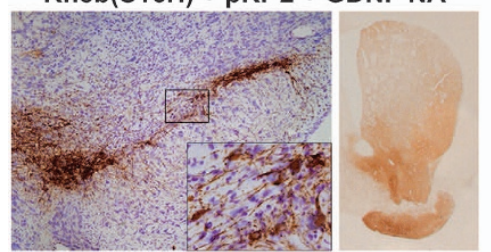

Rheb(S16H) + pKr-2 + BDNF NA

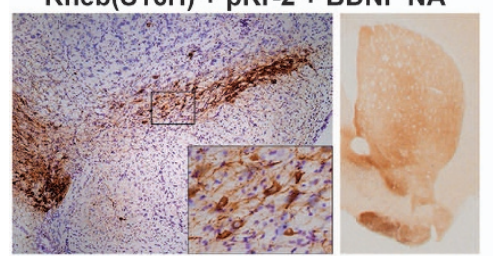

Rheb(S16H) + pKr-2 + Mix NA

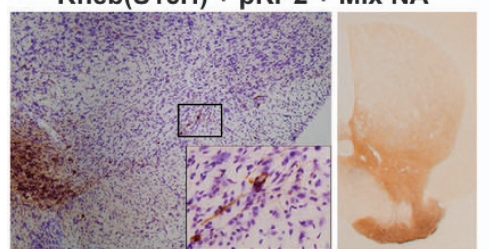

b

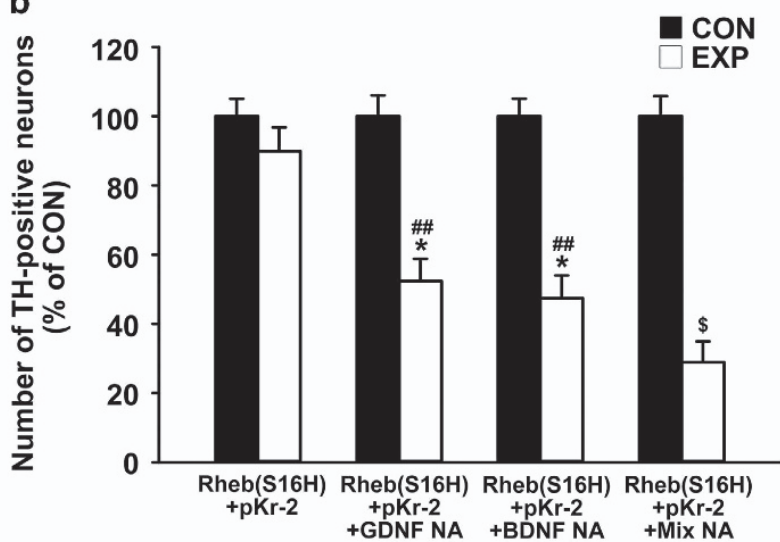

C

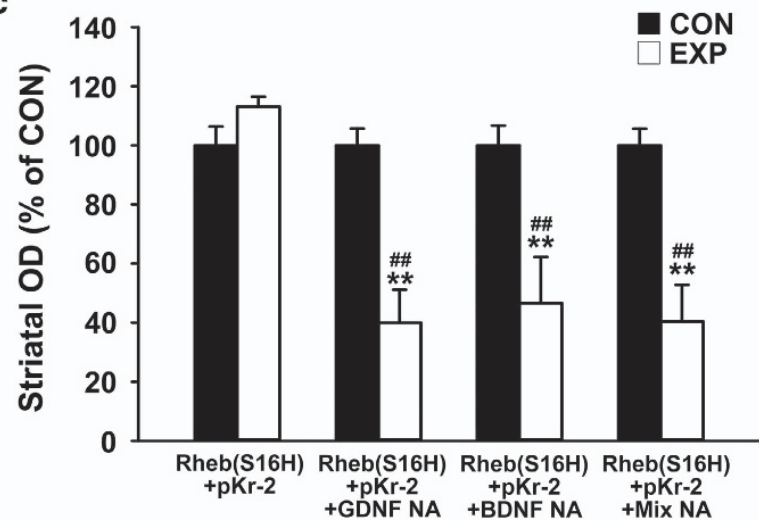

Figure 6 Upregulation of Rheb(S16H)-induced GDNF and BDNF contributes to protection of the nigrostriatal DA projection against pKr-2induced neurotoxicity in vivo. (a) Representative coronal sections of the SN and striatum following TH immunostaining. Scale bars, 200 and $500 \mu \mathrm{m}$ in the SN and striatum, respectively. The small panels show higher magnifications of the SN. Scale bar, $50 \mu \mathrm{m}$. Quantification of TH-positive neurons at 7 days after injection of pKr-2 and neutralizing antibodies against GDNF, BDNF, and Mix (BDNF + GDNF) in the presence of Rheb(S16H) and quantitative determination of striatal TH immunostaining. (b) TH-positive neurons in the counting area of the ipsilateral SN expressed as a percentage of those in the contralateral controls. CON, contralateral control side; EXP, ipsilateral injected side. ${ }^{*} P<0.001$ vs contralateral controls, $\# \# P<0.01$ vs $p K r-2$ alone in the presence of Rheb(S16H), $\$ P<0.01$ vs pKr2 and GDNF neutralizing antibody in the presence of Rheb(S16H) (one-way ANOVA and Tukey's post hoc analysis; $n=5$, each experimental group). (c) Optical density of TH-positive fibers at 7 days after injection of pKr-2 and neutralizing antibodies against GDNF, BDNF and Mix in the presence of Rheb(S16H). ${ }^{* *} P<0.01$ vs contralateral controls, ${ }^{\#} P<0.01$ vs pKr-2 alone in the presence of Rheb $(\mathrm{S} 16 \mathrm{H})$ (one-way ANOVA and Tukey's post hoc analysis; $n=5$, each experimental group). All values are expressed as the mean \pm s.e.m.

be useful for protecting nigral DA neurons in the adult brain. Numerous studies have focused on the mechanisms of neurotoxic inflammation inhibition that are involved in activated microglia and have shown that their control could result in the neuroprotection of nigral DA neurons. ${ }^{5-9,25-27,32}$ However, whether there is a specific treatment that can induce neuroprotective effects without the control of neuroinflammation in a neurotoxic inflammatory environment, thereby providing direct protection against neurotoxic inflammation in the adult brain, is still unknown.

The cellular effects of GDNF and BDNF are mainly initiated by their binding to specific receptors, such as GFR $\alpha 1-\mathrm{RET}^{33}$ and $\operatorname{TrkB},{ }^{13}$ respectively, and the actions of GDNF and BDNF, resulting in the downstream activation of $\mathrm{mTORC1}$, are crucial for neuronal survival and maintenance of the neuronal system. ${ }^{11-13,34-36}$ Moreover, consistent with the decreased levels of GDNF and BDNF in PD brains, ${ }^{10}$ a decrease in phosphorylated Akt, resulting in a loss of mTORC1 activation, was observed in the SN of patients with PD and a neurotoxin model of $\mathrm{PD},{ }^{37}$ and the upregulation of GDNF and BDNF could induce neuroprotective effects against neurodegenerative diseases such as Alzheimer's disease and PD. ${ }^{11-14,34}$ These results indicate that the support of neurotrophic factors and activation of the mTORC1 signaling pathway are necessary for the survival of DA neurons and the functional maintenance of the DA system in the adult brain. However, the systemic delivery of neurotrophic factors, such as GDNF and BDNF, as therapeutic agents is blocked by the blood-brain barrier, and their injection into the brain is not appropriate owing to their short half-life, despite their protective effects against DA neuron degeneration. ${ }^{15,16}$ Thus, the development of novel strategies to produce neurotrophic factors in the targeted region may be required for $\mathrm{PD}$ therapy, and a specific gene delivery system that can induce the persistent production of 
neurotrophic factors and activation of the mTORC1 signaling pathway in DA neurons can be utilized as a useful strategy against neurodegeneration in the nigrostriatal DA system in vivo.

To develop a novel strategy for sustained activation of the mTORC1 signaling pathway in adult neurons, we recently investigated the protective effects of AAV1 transduction of murine nigral DA neurons with constitutively active Rheb, which is a GTP-binding protein that directly activates mTORC1 to induce multiple cellular functions such as protein synthesis, cell growth, proliferation, survival, and synaptic plasticity, against neurotoxicity in animal models of PD. ${ }^{15,16,18,19}$ Our previous results demonstrated that Rheb $(\mathrm{S} 16 \mathrm{H})$ transduction in the $\mathrm{SN}$ could induce the sustained production of neurotrophic factors, such as GDNF and BDNF, via activation of the mTORC1 signaling pathway in Rheb $(\mathrm{S} 16 \mathrm{H})$-transduced DA neurons, which contributed to the protection of the nigrostriatal DA projection in neurotoxintreated animal models of PD. ${ }^{15,18}$ These results suggest that the activation of neuronal signaling pathways involved in cell survival by delivery of a specific gene such as Rheb(S16H) may be a useful strategy to protect the nigrostriatal DA system in the adult brain. However, whether the induction of neuronal mTORC1 activation by a specific gene delivery has neuroprotective resistance against neurotoxic inflammatory environments beyond the protective effects against intracellular neurotoxicity is still unclear.

Similar to previous studies, ${ }^{15,16,18,19}$ the immunohistochemical staining results demonstrated that AAV1-GFP and AAV1$\mathrm{Rheb}(\mathrm{S} 16 \mathrm{H})$ transduction in the SN could induce the expression of target proteins in adult neurons, including nigral DA neurons, with no expression in glial cells (Figure 1). However, despite the neuron-specific transduction of the viral vectors, our observations showed that neuronal expression of Rheb $(\mathrm{S} 16 \mathrm{H})$ protected the nigrostriatal DA projections against $\mathrm{pKr}-$ 2-induced neurotoxicity, potentially mediated by activated microglia, ${ }^{9,27}$ as demonstrated by immunohistochemical staining and western blot analysis for $\mathrm{TH}$ (Figure 2). Moreover, there was a significant protection of $\mathrm{TH}$-positive axons within the $\mathrm{MFB}$ of $\mathrm{Rheb}(\mathrm{S} 16 \mathrm{H})$-treated mice compared to that in mice treated with $\mathrm{pKr}-2$ alone (Figure $2 \mathrm{f}$ and $\mathrm{g}$ ), and a significant preservation of dopamine and its metabolites was also observed in $\mathrm{Rheb}(\mathrm{S} 16 \mathrm{H})$-treated mice with $\mathrm{pKr}$-2-induced neurotoxicity (Figure $3 \mathrm{a}-\mathrm{c}$ ). Consistent with these results, the behavioral test using the rota-rod apparatus showed that Rheb $(\mathrm{S} 16 \mathrm{H})$ transduction of DA neurons could protect locomotor activity, which is associated with the nigrostriatal DA system, ${ }^{38,39}$ from $\mathrm{pKr}$-2-induced neurotoxicity (Figure $3 \mathrm{~d}$ ). To clarify whether the neuroprotective effects of Rheb $(\mathrm{S} 16 \mathrm{H})$ transduction were through a resistance to $\mathrm{pKr}$-2-induced neuroinflammation or direct inhibition of microglial activation, we examined the levels of pro-inflammatory cytokines, such as TNF- $\alpha$ and IL- $1 \beta$, following pKr- 2 treatment in the absence/ presence of Rheb(S16H). Our results showed that there was no significant change in the expression levels of TNF- $\alpha$ and IL- $1 \beta$ in the Rheb(S16H)-treated SN compared to the levels in the SN treated with $\mathrm{pKr}-2$ alone (Figure 4), indicating that microglia activated by treatment with $\mathrm{pKr}-2$ caused similar neurotoxic inflammatory reactions in the Rheb(S16H)-treated SN. Therefore, these results suggest that the induction of Rheb $(\mathrm{S} 16 \mathrm{H})$ expression in DA neurons exerts its neuroprotective effects by providing a resistance to the neurotoxic inflammatory environment mediated by activated microglia.

To investigate how Rheb(S16H)-transduced DA neurons could have protective effects against neurotoxic inflammation induced by $\mathrm{pKr}-2$ treatment, we next assessed the expression levels of p-4E-BP1, indicating mTORC1 activation, and neurotrophic factors, such as GDNF and BDNF, which could be increased in nigral DA neurons by AAV1-Rheb $(\mathrm{S} 16 \mathrm{H})$ transduction in the $\mathrm{SN},{ }^{15,16,18,19}$ following $\mathrm{pKr}-2$ treatment in the absence or presence of $\operatorname{Rheb}(\mathrm{S} 16 \mathrm{H})$. Similar to a previous study, ${ }^{15}$ our results showed that the expression levels of $\mathrm{p}-4 \mathrm{E}$ BP1, GDNF and BDNF were significantly higher in the Rheb $(\mathrm{S} 16 \mathrm{H})$-transduced DA neurons than in the contralateral controls (Figure 4a), and the increases in p-4E-BP1 and both neurotrophic factors were significantly preserved at post-lesion day 1 following $\mathrm{pKr}-2$ treatment (Figure 5b). In addition, the levels of p-4E-BP1, GDNF and BDNF in the Rheb(S16H)transduced SN were maintained at levels similar to those in the contralateral controls at post-lesion day 7 , even though there were significant decreases in the $\mathrm{SN}$ following $\mathrm{pKr}-2$ treatment in the absence of Rheb(S16H) (Figure 5c). Moreover, our observations regarding the effects of neutralizing antibodies against GDNF and BDNF on Rheb(S16H)-induced neuroprotection indicated that neutralization of both neurotrophic factors could significantly reduce the protection of nigrostriatal DA projections from $\mathrm{pKr}$-2-induced neurotoxicity (Figure 6). However, whether the increases in BDNF and GDNF following Rheb $(\mathrm{S} 16 \mathrm{H})$ administration could intensify the activation of neurotrophic signaling pathways was unclear. Thus, to resolve this issue, we examined whether there were any changes in the levels of GDNF, BDNF and their receptors ${ }^{40,41}$ (Supplementary Figure S2) following treatment with neutralizing antibodies against GDNF and BDNF. Our results showed that the increases in GDNF and BDNF induced by Rheb(S16H) transduction were significantly attenuated by treatment with anti-GDNF and anti-BDNF, respectively (Supplementary Figure S2) and that co-treatment with both neutralizing antibodies induced synergistic effects to inhibit the activation of the Rheb(S16H)/mTORC1 signaling pathway (Supplementary Figure S2). Previous reports have shown that the expression and activation of neurotrophic factors could be regulated by a self-amplifying autocrine/paracrine action, which is one of the regulatory mechanisms of neurotrophic factors. ${ }^{42,43}$ Moreover, neutralizing antibodies against specific factors can act by binding to a ligand, resulting in inhibiting the biological action of the ligand through blocking it from interacting with its receptor. ${ }^{44}$ Therefore, the present results suggest that AAV1-Rheb $(\mathrm{S} 16 \mathrm{H})$ transduction induces an increase in neurotrophic factor production in DA neurons, and the produced trophic factors may also contribute to the 
activation of mTORC1 following Rheb(S16H) administration, consequently resulting in intensified neuroprotective effects.

In conclusion, we found that the robust trophic effects observed in nigral DA neurons induced by AAV1 transduction with $\mathrm{Rheb}(\mathrm{S} 16 \mathrm{H})$, which were previously reported in animal models of PD, ${ }^{15,16,18,19}$ remained under the neurotoxic inflammatory environment induced by $\mathrm{pKr}-2$ administration; ${ }^{9,25}$ those neurotrophic effects could offer promise for protecting the nigrostriatal DA projection from activated microglia that induce neurotoxic inflammatory events in the adult brain. Therefore, the present observations suggest that mTORC1 activation via a specific gene delivery to nigral DA neurons may be a useful strategy for protecting neurons against neurotoxic inflammation in the adult brain.

\section{CONFLICT OF INTEREST}

The authors declare no conflict of interest.

\section{ACKNOWLEDGEMENTS}

This study was supported by grants from the Korea Healthcare Technology R\&D Project, Ministry of Health \& Welfare (HI15C1928 and HI16C2210), the National Research Foundation of Korea (NRF-2014R1A1A2056508 and 2017R1A2B4002675) and the Korea Health Technology R\&D Project through the Korea Health Industry Development Institute (KHIDI; HI14C1135), funded by the Korean government.

\section{PUBLISHER'S NOTE}

Springer Nature remains neutral with regard to jurisdictional claims in published maps and institutional affiliations.

1 Dauer W, Przedborski S. Parkinson's disease: mechanisms and models. Neuron 2003; 39: 889-909.

2 Savitt JM, Dawson VL, Dawson TM. Diagnosis and treatment of Parkinson disease: molecules to medicine. J Clin Invest 2006; 116: 1744-1754.

3 Rascol O, Payoux P, Ory F, Ferreira JJ, Brefel-Courbon C, Montastruc JL. Limitations of current Parkinson's disease therapy. Ann Neurol 2003; 53 (Suppl 3): S3-12 discussion S12-15.

4 Pezzoli G, Zini M. Levodopa in Parkinson's disease: from the past to the future. Expert Opin Pharmacother 2010; 11: 627-635.

5 Block ML, Hong JS. Chronic microglial activation and progressive dopaminergic neurotoxicity. Biochem Soc Trans 2007; 35: 1127-1132.

6 Hirsch EC, Hunot S. Neuroinflammation in Parkinson's disease: a target for neuroprotection? Lancet Neurol 2009; 8: 382-397.

7 Magalingam KB, Radhakrishnan AK, Haleagrahara N. Protective mechanisms of flavonoids in Parkinson's disease. Oxid Med Cell Longev 2015; 2015: 314560

$8 \mathrm{Kim} \mathrm{HD}$, Jeong KH, Jung UJ, Kim SR. Naringin treatment induces neuroprotective effects in a mouse model of Parkinson's disease in vivo, but not enough to restore the lesioned dopaminergic system. J Nutr Biochem 2016; 28: 140-146.

9 Shin WH, Jeon MT, Leem E, Won SY, Jeong KH, Park SJ et al. Induction of microglial toll-like receptor 4 by prothrombin kringle-2: a potential pathogenic mechanism in Parkinson's disease. Sci Rep 2015; 5: 14764.

10 Chauhan NB, Siegel GJ, Lee JM. Depletion of glial cell line-derived neurotrophic factor in substantia nigra neurons of Parkinson's disease brain. J Chem Neuroanat 2001; 21: 277-288.

11 Allen SJ, Watson JJ, Shoemark DK, Barua NU, Patel NK. GDNF, NGF and BDNF as therapeutic options for neurodegeneration. Pharmacol Ther 2013; 138: 155-175.

12 Lin LF, Doherty DH, Lile JD, Bektesh S, Collins F. GDNF: a glial cell linederived neurotrophic factor for midbrain dopaminergic neurons. Science 1993; 260: 1130-1132.
13 Manfredsson FP, Okun MS, Mandel RJ. Gene therapy for neurological disorders: challenges and future prospects for the use of growth factors for the treatment of Parkinson's disease. Curr Gene Ther 2009; 9: 375-388.

14 Hyman C, Hofer M, Barde YA, Juhasz M, Yancopoulos GD, Squinto SP et al. BDNF is a neurotrophic factor for dopaminergic neurons of the substantia nigra. Nature 1991; 350: 230-232.

$15 \mathrm{Nam}$ JH, Leem E, Jeon MT, Jeong KH, Park JW, Jung UJ et al. Induction of GDNF and BDNF by hRheb(S16H) transduction of SNpc neurons: neuroprotective mechanisms of hRheb(S16H) in a model of Parkinson's disease. Mol Neurobiol 2015; 51: 487-499.

16 Jeong KH, Nam JH, Jin BK, Kim SR. Activation of CNTF/CNTFRalpha signaling pathway by hRheb(S16H) transduction of dopaminergic neurons in vivo. PLOS ONE 2015; 10: e0121803.

17 Peterson AL, Nutt JG. Treatment of Parkinson's disease with trophic factors. Neurotherapeutics 2008; 5: 270-280.

18 Kim SR, Kareva T, Yarygina O, Kholodilov N, Burke RE. AAV transduction of dopamine neurons with constitutively active Rheb protects from neurodegeneration and mediates axon regrowth. Mol Ther 2012; 20: 275-286.

19 Kim SR, Chen X, Oo TF, Kareva T, Yarygina O, Wang C et al. Dopaminergic pathway reconstruction by Akt/Rheb-induced axon regeneration. Ann Neurol 2011; 70: 110-120.

20 Li Y, Hu X, Liu Y, Bao Y, An L. Nimodipine protects dopaminergic neurons against inflammation-mediated degeneration through inhibition of microglial activation. Neuropharmacology 2009; 56: 580-589.

21 Chung YC, Kim SR, Park JY, Chung ES, Park KW, Won SY et al. Fluoxetine prevents MPTP-induced loss of dopaminergic neurons by inhibiting microglial activation. Neuropharmacology 2011; 60: 963-974.

22 Jeon MT, Nam JH, Shin WH, Leem E, Jeong KH, Jung UJ et al. In vivo AAV1 transduction with $\mathrm{hRheb}(\mathrm{S} 16 \mathrm{H})$ protects hippocampal neurons by BDNF production. Mol Ther 2015; 23: 445-455.

23 Kurauchi Y, Hisatsune A, Isohama Y, Mishima S, Katsuki H. Caffeic acid phenethyl ester protects nigral dopaminergic neurons via dual mechanisms involving haem oxygenase-1 and brain-derived neurotrophic factor. $\mathrm{Br} J$ Pharmacol 2012; 166: 1151-1168.

24 Kim BW, Jeong KH, Kim JH, Jin M, Kim JH, Lee MG et al. Pathogenic upregulation of glial lipocalin-2 in the Parkinsonian dopaminergic system. $J$ Neurosci 2016; 36: 5608-5622.

25 Kim SR, Chung ES, Bok E, Baik HH, Chung YC, Won SY et al. Prothrombin kringle-2 induces death of mesencephalic dopaminergic neurons in vivo and in vitro via microglial activation. J Neurosci Res 2010; 88: 1537-1548.

$26 \mathrm{Nam}$ JH, Leem E, Jeon MT, Kim YJ, Jung UJ, Choi MS et al. Inhibition of prothrombin kringle-2-induced inflammation by minocycline protects dopaminergic neurons in the substantia nigra in vivo. Neuroreport 2014; 25: 489-495.

27 Leem E, Jeong KH, Won SY, Shin WH, Kim SR. Prothrombin Kringle-2: a potential inflammatory pathogen in the Parkinsonian dopaminergic system. Exp Neurobiol 2016; 25: 147-155.

$28 \mathrm{McGeer} \mathrm{PL}$, Itagaki S, Boyes BE, McGeer EG. Reactive microglia are positive for HLA-DR in the substantia nigra of Parkinson's and Alzheimer's disease brains. Neurology 1988; 38: 1285-1291.

29 Akiyama H, McGeer PL. Microglial response to 6-hydroxydopamine-induced substantia nigra lesions. Brain Res 1989; 489: 247-253.

30 Czlonkowska A, Kohutnicka M, Kurkowska-Jastrzebska I, Czlonkowski A. Microglial reaction in MPTP (1-methyl-4-phenyl-1,2,3,6-tetrahydropyridine) induced Parkinson's disease mice model. Neurodegeneration 1996; 5: 137-143.

31 Sherer TB, Betarbet R, Kim JH, Greenamyre JT. Selective microglial activation in the rat rotenone model of Parkinson's disease. Neurosci Lett 2003; 341: 87-90.

32 Choi I, Kim B, Byun JW, Baik SH, Huh YH, Kim JH et al. LRRK2 G2019S mutation attenuates microglial motility by inhibiting focal adhesion kinase. Nat Commun 2015; 6: 8255.

33 Drinkut A, Tillack K, Meka DP, Schulz JB, Kugler S, Kramer ER. Ret is essential to mediate GDNF's neuroprotective and neuroregenerative effect in a Parkinson disease mouse model. Cell Death Dis 2016; 7: e2359.

34 Siegel GJ, Chauhan NB. Neurotrophic factors in Alzheimer's and Parkinson's disease brain. Brain Res Brain Res Rev 2000; 33: 199-227.

35 Howells DW, Porritt MJ, Wong JY, Batchelor PE, Kalnins R, Hughes AJ et al. Reduced BDNF mRNA expression in the Parkinson's disease substantia nigra. Exp Neurol 2000; 166: 127-135.

36 Studer L, Spenger C, Seiler RW, Othberg A, Lindvall O, Odin P. Effects of brain-derived neurotrophic factor on neuronal structure of dopaminergic neurons in dissociated cultures of human fetal mesencephalon. Exp Brain Res 1996; 108: 328-336. 
37 Selvaraj S, Sun Y, Watt JA, Wang S, Lei S, Birnbaumer L et al. Neurotoxininduced ER stress in mouse dopaminergic neurons involves downregulation of TRPC1 and inhibition of AKT/mTOR signaling. J Clin Invest 2012; 122: 1354-1367.

38 Beninger RJ. The role of dopamine in locomotor activity and learning. Brain Res 1983; 287: 173-196.

39 Ryczko D, Dubuc R. Dopamine and the brainstem locomotor networks: from lamprey to human. Front Neurosci 2017; 11: 295.

40 deGroot JW, Links TP, Plukker JT, Lips CJ, Hofstra RM. RET as a diagnostic and therapeutic target in sporadic and hereditary endocrine tumors. Endocr Rev 2006; 27: 535-560.

41 Minichiello L. TrkB signalling pathways in LTP and learning. Nat Rev Neurosci 2009; 10: 850-860.

42 Cheng PL, Song AH, Wong YH, Wang S, Zhang X, Poo MM. Self-amplifying autocrine actions of BDNF in axon development. Proc Natl Acad Sci USA 2011; 108: 18430-18435.

43 Barak S, Ahmadiantehrani S, Kharazia V, Ron D. Positive autoregulation of GDNF levels in the ventral tegmental area mediates long-lasting inhibition of excessive alcohol consumption. Trans/ Psychiatry 2011; 1, e60.
44 Bradbury AR, Sidhu S, Dubel S, McCafferty J. Beyond natural antibodies: the power of in vitro display technologies. Nat Biotechnol 2011; 29: 245-254.

cc) (i) $(\Theta)$ This work is licensed under a Creative Commons Attribution-NonCommercial-NoDerivs

International License. The images or other third party material in this article are included in the article's Creative Commons license, unless indicated otherwise in the credit line; if the material is not included under the Creative Commons license, users will need to obtain permission from the license holder to reproduce the material. To view a copy of this license, visit http://creativecommons.org/licenses/by-nc-nd/4.0/

(C) The Author(s) 2018

Supplementary Information accompanies the paper on Experimental \& Molecular Medicine website (http://www.nature.com/emm) 